\title{
Sistemas de Recomendação de Recursos Educacionais para Grupos de Redes Sociais: um Mapeamento Sistemático
}

\author{
Rafael F. Almeida, Fernanda Campos, José Maria N. David, Victor Stroele \\ Universidade Federal de Juiz de Fora (UFJF) - Programa de Pós-graduação em Ciência \\ da Computação - Núcleo de Pesquisa em Engenharia do Conhecimento \\ Juiz de Fora - MG - Brasil \\ rafaelfalmeida@gmail.com, fernanda.campos@ufjf.edu.br, \\ jmndavidegmail.com, victor.stroeledice.ufjf.br
}

\begin{abstract}
Resumo. Sistemas de recomendação têm atraído a atenção de acadêmicos e profissionais. O grande desafio é a escolha adequada de recursos educacionais distribuidos em diferentes repositórios. Considerando que os alunos representam grupos de usuários com características comuns, apresenta-se um mapeamento sistemático, onde foram identificados 166 artigos de pesquisa sobre sistemas de recomendação para grupos de usuário em redes sociais, com foco na recomendação de recursos educacionais, publicados entre 2004 e 2014. O objetivo é identificar tendências em pesquisas e características para grupos de usuários em redes sociais.
\end{abstract}

\begin{abstract}
Recommender systems have attracted the attention of researchers and professionals. The great challenge is to choose the proper educational resource distributed in different repositories. Considering that studentres represent users group with common features, this study performs a systematic mapping, where 166 research articles were identified, about recommendation for user groups in social networks, focusing on the recommendation of educational resources, published between 2004 and 2014. The main goal is to understand the trend of research and features of users groups in social networks.
\end{abstract}

\section{Introdução}

Sistemas de Recomendação (SR) tornaram-se uma importante área de pesquisa desde meados da década de 1990 (Kim et al., 2010). Eles coletam informações sobre as preferências de seus usuários para um conjunto de itens (filmes, músicas, livros, gadgets, aplicativos, sites, destinos de viagens, material de e-learning, entre outros). Em geral, os SR ajudam diretamente os usuários a encontrar conteúdos, produtos ou serviços (Park et al., 2012).

Os SR são grandes aliados da personalização de sistemas computacionais, principalmente na web, sendo capazes de identificar preferências e sugerir itens relevantes para cada usuário, de acordo com a análise de seu comportamento de navegação, consulta e/ou compra, preferências, entre outros aspectos (Cazella et al. 2012). SR são de particular importância em ambientes sociais, onde os usuários compartilham acesso a um conjunto comum de recursos. A variabilidade das características de um usuário, como sua origem, seus interesses especiais e seu grau de especialização, é usada para sugerir itens interessantes, úteis e compreensíveis para um usuário específico (Ribeiro et al., 2013). 
Existem inúmeros recursos educacionais (materiais de ensino em qualquer suporte ou mídia) distribuídos em diferentes repositórios, que abordam um conjunto amplo de assuntos e que possuem objetivos educacionais distintos. Em virtude disso, a escolha adequada desses recursos é um desafio para os educadores e para os próprios alunos. Nesse cenário, um SR tem um papel importante para auxiliar educadores e alunos a encontrarem recursos educacionais relevantes e pertinentes aos seus perfis e ao contexto em que estão inseridos (Pereira et al., 2014).

Com base na necessidade de compreender melhor a área e identificar as deficiências da mesma, este trabalho tem o objetivo de identificar estudos sobre SRs através de um mapeamento sistemático, buscando elementos que caracterizam o cenário de recomendação de recursos educacionais para grupos em redes sociais. Com isso, a proposta é organizar os trabalhos encontrados nesta revisão, disponibilizando uma rápida referência aos desenvolvedores e pesquisadores da área, e identificar oportunidades para pesquisas futuras.

Este trabalho está organizado da seguinte forma: a seção 2 apresenta a metodologia de pesquisa, cujos passos são definidos no protocolo de pesquisa para o mapeamento sistemático. A seção 3 apresenta os dados coletados com informações gerais sobre os estudos selecionados. Já a seção 4 descreve os resultados do mapeamento sistemático. A seção 5 apresenta as ameaças à validade do trabalho e, finalmente, a seção 6 apresenta as considerações finais, juntamente com a análise dos resultados e principais contribuições.

\section{Metodologia de pesquisa}

A atividade de planejamento inclui a identificação dos objetivos e a definição de um protocolo. O protocolo especifica o método a ser utilizado para realizar a revisão sistemática (Kitchenham, 2004). As revisões sistemáticas são, portanto, passíveis de auditoria e replicáveis, no sentido de que outros profissionais podem reproduzir o mesmo protocolo e julgar a adequação dos padrões escolhidos. Esta seção apresenta o método de estudo de mapeamento sistemático de pesquisa. Este estudo foi realizado de junho a setembro de 2014 e revisado de abril a maio de 2015 .

\subsection{Mapeamento sistemático}

Estudos de mapeamento são projetados para fornecer uma visão geral de uma área de pesquisa, fornecendo uma indicação sobre a quantidade e o tipo de pesquisa disponível dentro dele. Assim, as questões relacionadas com mapeamento estão preocupadas com a estrutura da área e as questões relacionadas com a revisão e com a evidência (Petersen, 2011). Este mapeamento sistemático foi elaborado para responder as seguintes questões:

- QM1: Como as publicações sobre SR estão distribuídas ao longo dos anos?

Esta questão pretende apontar o crescimento ou a redução da área de pesquisa.

- QM2: Quais são os autores mais ativos na área?

Com esta pergunta, pretende-se ter uma indicação dos principais pesquisadores da área, oferecendo uma referência para publicações relacionadas em pesquisas futuras.

- QM3: Quais meios de publicações se interessam por SR?

Ao responder a esta pergunta, espera-se identificar onde os artigos podem ser encontrados, bem como identificar oportunidades para a publicação de estudos futuros. 
Já a revisão sistemática da literatura visa identificar, avaliar e interpretar todas as pesquisas disponíveis relevantes para uma questão de pesquisa específica, ou área temática, ou fenômeno de interesse. Formular as questões da pesquisa é a atividade mais importante durante a definição do protocolo (Kitchenham, 2004). Esta pesquisa sistemática foi elaborada para responder a uma pergunta principal de pesquisa e três questões de pesquisa secundárias:

- Como SR podem recomendar recursos educacionais para grupos de usuários de redes sociais?

○ QP1: Qual abordagem de recomendação é mais utilizada para grupos de usuários em redes sociais?

Os SR podem ser classificados em três grupos quanto à sua abordagem: Filtragem Baseada em Conteúdo (FBC), Filtragem Colaborativa (FC) e Filtragem Híbrida (FH) (Casagrande et al., 2013). Esta questão pretende identificar a abordagem mais comumente utilizada no desenvolvimento dos SR para grupos de usuários em redes sociais, a fim de auxiliar na escolha da utilização de uma abordagem em trabalhos futuros.

○ QP2: Quais recursos educacionais são mais abordados por SR em grupo nas redes sociais?

Esta questão pretende responder em quais tipos de mídias de recursos educacionais os usuários teriam um maior interesse pelo recurso educacional recomendado. Com isso, pode-se focar a recomendação em alguns tipos como vídeos, textos ou sons.

○ QP3: Quais as principais técnicas de extração de dados dos grupos de usuários estão sendo abordadas?

Esta questão pretende oferecer indícios para a escolha de uma técnica de extração de dados em trabalhos futuros. O objetivo é verificar quais técnicas vem sendo abordadas na extração de dados de grupos de usuários em redes sociais, a fim de permitir definir a construção de um perfil para o grupo, e não apenas ao usuário membro do grupo.

\subsection{Termos de busca}

As questões de pesquisa conduzem à definição de uma consulta a ser executada em repositórios científicos. Para compor esta consulta, palavras chave são definidas. A consulta apresenta um conjunto de expressões que combinam palavras chave e seus sinônimos, dispostos de forma que aumente o número de artigos relevantes obtidos a partir da pesquisa (Petersen, 2011).

Os termos de pesquisa são construídos em três etapas: estruturação de questões de pesquisa em termos de PICOC (População, Intervenção, Comparação, Saída, e Contexto) (Kitchenham e Charters, 2007), a fim de identificar palavras-chave, a identificação de sinônimos para cada uma das palavras-chave e construir a sequência de pesquisa com base na combinação dos termos-chave e seus sinônimos, usando os operadores OR e AND. Comparação não é relevante neste trabalho, uma vez que este mapeamento visa conceber uma visão geral do assunto através de um estudo exploratório. O resultado deste processo é apresentado na Tabela 1.

Tabela 1. Termos de busca

\begin{tabular}{|l|l|}
\hline PICOC & Palavras-chave \\
\hline Population & $\begin{array}{l}\text { "social group network", "social group application", "social group } \\
\text { knowledge", "group user profile" }\end{array}$ \\
\hline Intervention & "recommender", "recommendation" \\
\hline Comparison & Notapplicable \\
\hline
\end{tabular}


CBIE-LACLO 2015

Anais do XXVI Simpósio Brasileiro de Informática na Educação (SBIE 2015)

\begin{tabular}{|l|l|}
\hline Outcome & $\begin{array}{l}\text { "system", "program", "approach", "tool", "software", "model”, } \\
\text { "process", "method", "technique", "educationalresource", } \\
\text { "educationalmaterial" }\end{array}$ \\
\hline Context & "educational", "education", "learning", "e-leaning"
\end{tabular}

A string de busca foi definida como uma combinação das palavras-chave de acordo com o PICOC, além da exibição dos artigos de controle, sendo respectivamente:

- Quijano-Sánchez, L., Díaz-Agudo, B., Recio-García, J. A. (2014) "Development of a group recommender application in a Social Network" in Knowledge-Based Systems.

- Fatemi, M., Tokarchuk, L. (2013) "A Community Based Social Recommender System for individuals \& groups", in: ASE/IEEE International Conference on Social Computing (SocialCom), Washington, DC, pp. 351-356.

- Rafaeli, S., Dan-Gur, Y., Barak, M. (2005) "Social recommender systems: Recommendations in support of E-learning" in International Journal of Distance Education Technologies, 3 (2), pp. 30-47.

A Tabela 2 apresenta a string gerada por esta estratégia. Ao efetuar a pesquisa com a string nas bases, foram incluídos trabalhos somente do domínio de pesquisa "Ciência da Computação". Além disso, a string de busca foi validada por uma especialista da área.

Tabela 2. String de busca derivada do PICOC

String de Busca

("social" OR "social network")AND ("group")AND ("recommender" OR "recommendation") AND ("system" OR "program" OR “approach" OR "tool” OR "software” OR "model” OR "process" OR "method" OR "technique")AND("learning" OR "e-learning" OR "educational" OR "education")

\subsection{Fonte de pesquisa}

A cadeia de consulta foi usada para recuperar os trabalhos candidatos nas seguintes fontes de busca, selecionados com base em estudos anteriores (Kitchenham et al., 2010; Prikladnicki e Audy, 2010), além de outras fontes de busca consideradas relevantes para a pesquisa:

- IEEE Digital Library (http://ieeexplore.ieee.org);

- ACM Digital Library (http://portal.acm.org);

- Springer Link (http://springerlink.com);

- Science@Direct (http://www.sciencedirect.com)

- Citeseer (http://citeseerx.ist.psu.edu);

- ISI Web of Science (www.isiknowledge.com);

- Scopus (http://www.scopus.com);

- DBLP (http://www.dblp.org/search/)

- El Compendex (http://www.engineeringvillage.com)

- Simpósio Brasileiro de Informática na Educação (Sbie);

- Congresso Brasileiro de Software (Cbsoft).

Em adição à utilização das fontes de busca, este mapeamento também realizou o snowballing (Jalali e Wohlin, 2012). Snowballing é uma técnica de engenharia de software baseada em evidências para encontrar obras relevantes com base em referências de estudos e em obras que realmente mencionam os estudos selecionados. A Scopus foi adotada para apoiar este processo. 


\subsection{Critérios de inclusão e exclusão}

Todos os documentos obtidos pela execução da string de busca em cada fonte selecionada foram analisados para verificar a sua relevância para esta pesquisa. O processo usado para incluir ou excluir um trabalho foi baseado em (Kitchenham e Charters, 2007) (Prikladnicki e Audy, 2010), e compreende os seguintes passos:

- CE1: Os trabalhos que não possuem informações básicas de autor ou título foram excluídos.

- CE2: Os documentos duplicados foram reunidos e tiveram suas repetições excluídas.

- CE3: Foram consideradas publicações entre 2004 e 2014. Este período de 10 anos foi considerado representativo para a pesquisa.

- CE4: Os títulos, resumos e palavras-chave dos artigos foram lidos, e os que foram consideradas claramente irrelevantes para as questões de pesquisa, foram excluídos.

\section{Coleta de dados}

Inicialmente, as pesquisas realizadas geraram uma lista contendo todos os documentos encontrados. Este processo foi concluído com a ajuda das ferramentas Zotero ${ }^{1}$ e Parsifal ${ }^{2}$. O Zotero permite, através de um plugin utilizado em um navegador, exportar as informações dos artigos encontrados através da string de busca para um arquivo no formato Bibtex. Esta funcionalidade é muito útil, visto que nem todas as bases de pesquisa possuem um mecanismo para exportar o resultado das buscas. Já o Parsifal permite selecionar o trabalho e marcá-lo como aceito, rejeitado ou duplicado, para cada base de pesquisa, e, também oferece suporte a todo o desenvolvimento do protocolo do mapemanto.

A busca nas bases do Sbie e Cbsoft foram feitas manualmente. Foram consideradas importantes para a pesquisa nestas bases os trabalhos a partir de 2008, quando começaram a surgir trilhas com o tema. Na fonte de pesquisa Springer, somente foram considerados os trabalhos disponibilizados para consulta, ou seja, os trabalhos bloqueados foram descartados. Logo após foram implantados os filtros de exclusão e inclusão, seguindo os critérios definidos anteriormente. A Tabela 3 mostra o número de estudos em cada etapa e por cada biblioteca digital.

Tabela 3. Bases de pesquisa

\begin{tabular}{|l|l|l|l|l|l|}
\hline & String & CE1 & CE2 & CE3 & CE4 \\
\hline IEEE & 50 & 50 & 35 & 33 & 16 \\
\hline ACM & 58 & 53 & 39 & 25 & 4 \\
\hline Springer & 95 & 94 & 92 & 80 & 24 \\
\hline Science Direct & 38 & 38 & 33 & 29 & 8 \\
\hline Citeseer & 18 & 16 & 13 & 6 & 4 \\
\hline ISI & 29 & 29 & 20 & 17 & 10 \\
\hline Scopus & 171 & 149 & 103 & 99 & 24 \\
\hline DBLP & 9 & 9 & 8 & 8 & 8 \\
\hline El Compendex & 227 & 195 & 142 & 140 & 36 \\
\hline Sbie & 28 & 28 & 28 & 28 & 11 \\
\hline Cbsoft & 5 & 5 & 5 & 5 & 0 \\
\hline TOTAL & $\mathbf{7 2 8}$ & $\mathbf{6 6 6}$ & $\mathbf{5 1 8}$ & $\mathbf{4 7 0}$ & $\mathbf{1 4 5}$ \\
\hline
\end{tabular}

${ }^{1}$ https://www.zotero.org/

${ }^{2} \mathrm{http}: / /$ parsif.al/ 
Com a finalidade de auxiliar o entendimento destes dados, a figura 1 mostra os trabalhos aceitos por fonte de pesquisa.

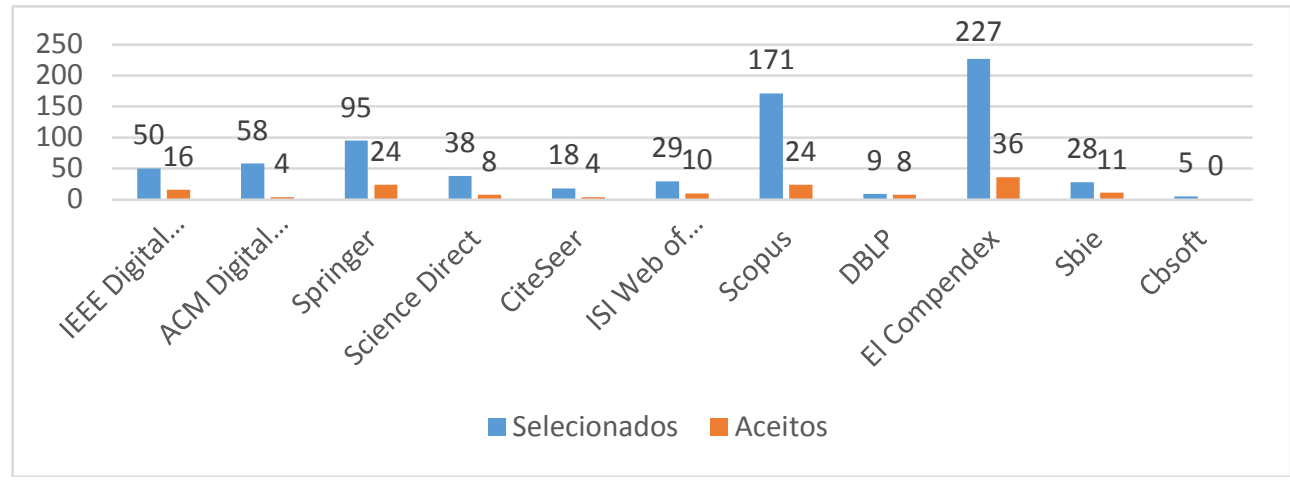

Figura 1. Artigos aceitos por fonte de pesquisa

O processo de seleção utilizando o método snowballing foi realizada de forma semelhante a partir do CE3. Depois de identificar os estudos, a leitura de seus títulos, resumos e palavras-chave a partir das referências, estudos relacionados, e as obras mais recentes que fazem referência desses estudos, foram adicionadas para análise das questões, sendo incluídos mais 21 trabalhos que não haviam sido identificados anteriormente, totalizando 166 trabalhos relevantes para o mapeamento sistemático. Além disso, as referências claramente irrelevantes foram imediatamente descartadas. A figura 2 mostra o número de estudos em cada etapa da filtragem de trabalhos com a aplicação do snowballing.
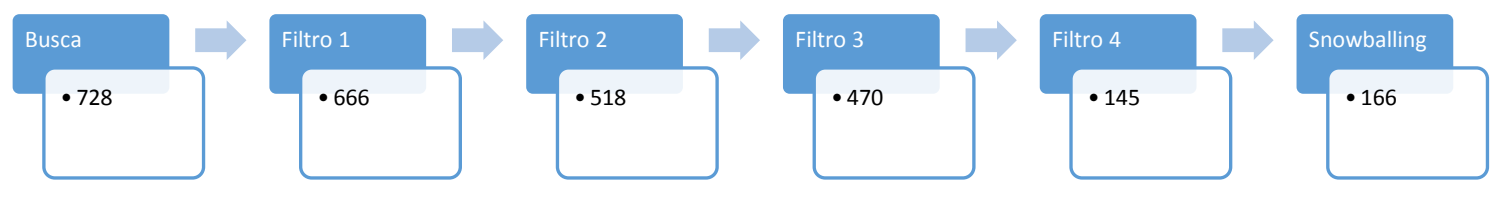

Figura2. Número de estudos por etapa da filtragem

\section{Relatório do mapeamento sistemático}

Um mapeamento sistemático tem como objetivo fornecer uma visão geral de uma área de pesquisa e identificar a quantidade e o tipo de pesquisa disponível dentro dele. Nesta seção, são apresentadas as respostas das questões de mapeamento.

Para responder a QM1 - Como as publicações sobre SR estão distribuídas ao longo dos anos? - a figura 3 é apresentada. Através dela, obtemos indícios de que a área vem sendo mais pesquisada nos últimos anos, com um crescente aumento no número de trabalhos relevantes, o que também evidencia a importância deste mapeamento sistemático, uma vez que a área foco do estudo é de um crescente interesse da comunidade.

Para responder a QM2 - Quais são os autores mais ativos na área? - a tabela 4 é apresentada. Nela são listados o nome e o número de trabalhos publicados dos autores considerados mais ativos. Os autores que tiveram o mesmo número de trabalhos selecionados foram ordenados de acordo com os trabalhos publicados mais recentemente. Este cenário é adequado ao interesse do mapeamento proposto, não sendo realidade em outros contextos específicos. 


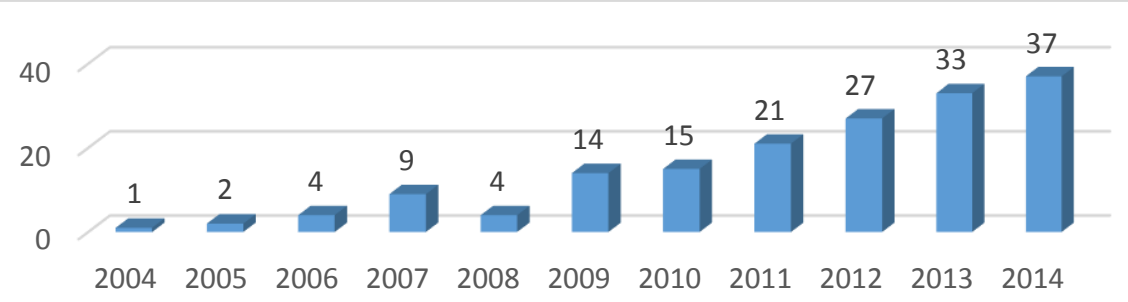

Figura 3. Número de trabalhos relevantes por ano

Tabela 4. Autores mais ativos

\begin{tabular}{|l|c|}
\hline Autor & Número de trabalhos \\
\hline Quijano-Sánchez, Lara & 6 \\
\hline Díaz-Agudo, Belén & 6 \\
\hline Recio-García, Juan A. & 6 \\
\hline Wang, Y.a & 4 \\
\hline Zhang, J.b & 3 \\
\hline Vassileva, J.a & 3 \\
\hline Wan, X.a & 3 \\
\hline Okamoto, T.a & 3 \\
\hline Ye, Mao & 3 \\
\hline
\end{tabular}

Para responder a QM3 - Quais os veículos de publicações se interessam por SR? - a Tabela 5 é apresentada. Nessa tabela são apresentados os meios de publicação dos trabalhos selecionados, com o número de publicações encontradas.

Tabela 5. Principais meios de publicações

\begin{tabular}{|l|l|}
\hline Veículo da publicação (Anais, periódicos, etc.) & Número de publicações \\
\hline User Modeling and User-Adapted Interaction & 19 \\
\hline RecSys - Proceedings of ACM Conference on Recommender Systems & 8 \\
\hline Lecture Notes in Computer Science & 7 \\
\hline CEUR Workshop Proceedings & 6 \\
\hline Simpósio Brasileiro de Informática na Educação & 6 \\
\hline Expert Systems withApplications & 4 \\
\hline Multimedia Systems & 4 \\
\hline
\end{tabular}

Para responder a QP1 - Qual abordagem de recomendação é mais utilizada para grupos de usuários em redes sociais? - Os SR foram classificados em três grupos: FBC, FC e FH. Sendo assim, foram identificados na figura 4 os trabalhos que claramente apresentam a abordagem utilizada, permitindo, através dela, responder a QP1. O maior número de trabalhos relacionados está utilizando a $\mathrm{FBC}$, porém não existem indícios que esta abordagem é melhor, ela somente vem sendo mais utilizada.

Para responder a QP2 - Quais os recursos educacionais são mais abordados por SR em grupo nas redes sociais? - foi preciso classificar as mídias encontradas em documento, imagem, livro, som e vídeo. Desta maneira, foram identificados na figura 5 os trabalhos que apresentam o recurso recomendado, permitindo, através dela, responder a QP2. Verifica-se que claramente usuários de redes sociais são abastecidos de recursos mais dinâmicos, onde a leitura acaba sendo utilizada de uma maneira reduzida. Os usuários tendem a ser recomendados com recursos de vídeo, som e imagem quase na sua totalidade de recomendações.

Para responder a QP3 - Quais as principais técnicas de extração de dados dos grupos de usuários estão sendo abordadas? - utilizou-se a classificação por técnicas de 
extração proposta por Park et al. (2012). Park et al. (2012) classifica as técnicas utilizadas para extração de dados em: association rule, clustering, decision tree, $k$-nearest neighbor $(k-n n)$, link analisys, neural network, regression e heuristic. Sendo assim, foram identificados na figura 6 os trabalhos que claramente apresentam a técnica de extração utilizada, permitindo, através dela, responder a QP3. Verifica-se que não existe alguma técnica que seja amplamente utilizada para o propósito desta revisão, ou seja, os trabalhos selecionados não tendem a trabalhar em uma técnica de extração específica ou não abordam como as informações estão sendo extraídas.

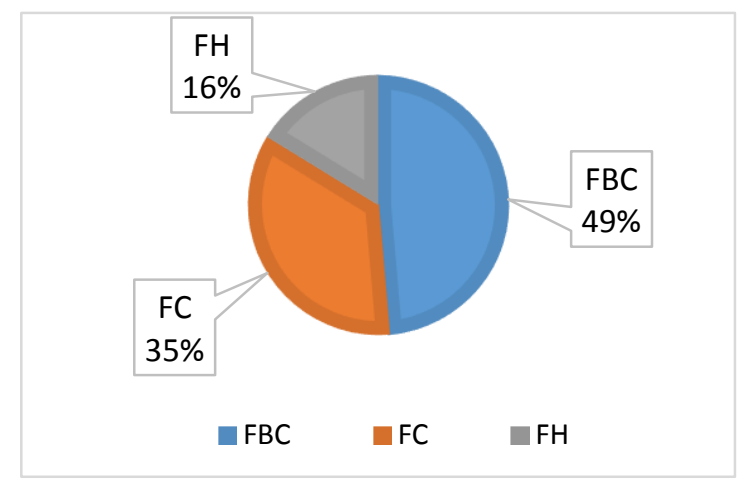

Figura 4. Abordagens de recomendação utilizadas

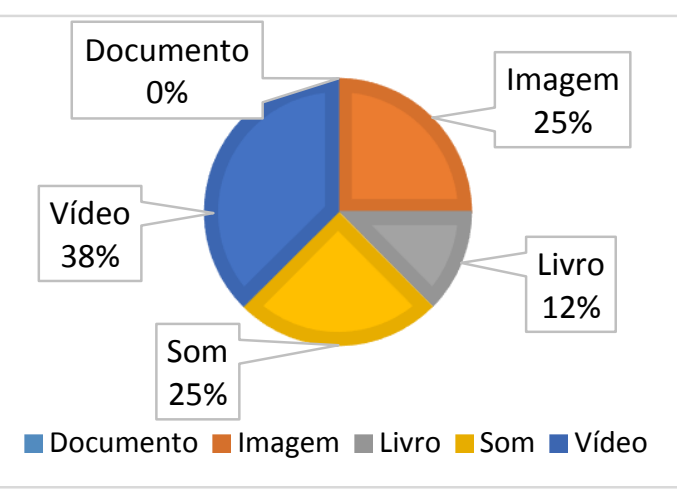

Figura 5. Recursos recomendados

Analisando as informações, podemos observar que há uma tendência no desenvolvimento de SR que usam uma abordagem FBC e recomendam recursos dinâmicos. Futuramente iremos desenvolver um SR que irá abordar recomendações em grupos de usuários em redes sociais.

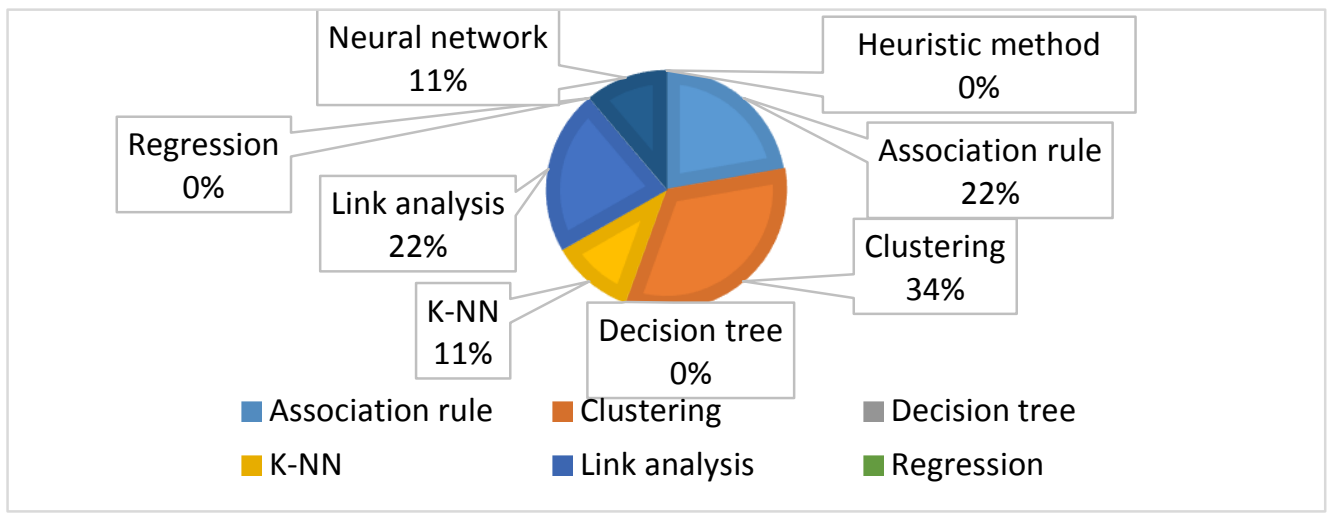

Figura 6. Técnicas de extração de dados utilizadas

\section{Ameaças a validade}

Existem algumas ameaças à validade do nosso estudo. Eles são apresentados abaixo com as estratégias para a sua mitigação. Quanto a string de busca, é possível que ele não tenha abordado alguns estudos. Para mitigar esta ameaça, uma especialista na área foi consultada a fim de validar a string, e também foi aplicada a técnica do snowballing com as referências dos estudos primários selecionados. Outra ameaça relacionada com a string de busca é que ela encontrou um grande número de trabalhos (728). Analisar esse grande 
número de trabalhos encontrados em um curto período de tempo ocasionou um grande esforço e a atividade pode ter sido influenciada por fadiga no processo.

Em relação ao viés de publicação, não se pode garantir que todos os estudos primários relevantes foram selecionados. Mesmo procurando nas principais bibliotecas digitais, é possível que alguns estudos relevantes não tenham sido incluídos no processo de busca. Como na string de busca, esta ameaça também foi mitigada realizando a técnica do snowballing, com as referências dos estudos primários. Além disso, outra estratégia utilizada para mitigar esta ameaça foi a utilização de um grande número de bibliotecas digitais.

Em relação à pesquisa realizada, os processos de busca nas bases de dados digitais não são exatamente os mesmos, e não é mostrado como elas funcionam internamente. Para mitigar esta ameaça, foram adaptadas as strings de busca para cada banco de dados digital, tomando cuidado para que as expressões lógicas se tornassem equivalentes de forma consistente em todos eles.

\section{Conclusão}

Recentemente redes sociais têm sido utilizadas em várias aplicações. No entanto, estudos sobre SR por meio de análise de rede social ainda são deficientes. Outra área pouco explorada é a recomendação de recursos educacionais em redes sociais. Portanto, o desenvolvimento da pesquisa em SR utilizando análise de redes sociais, bem como a recomendação de recursos educacionais nas redes sociais são áreas interessantes de pesquisa.

SR que consideram grupos de usuários estão começando a se expandir e para ser usado em diferentes áreas. Os resultados apresentados neste documento têm diversas implicações importantes. Com base nas taxas de publicações, percebe-se que o interesse em SR vem crescendo constantemente e significativamente. As recomendações em grupo geram sugestões com capacidade de satisfazer um grupo de usuários com interesses potencialmente comuns.

Tendo como base este estudo pretende-se propor uma arquitetura capaz de extrair informações acerca do interesse educacional e informações básicas de grupos utilizando as redes sociais e os ambientes virtuais de aprendizagem, determinando o perfil educacional do grupo a partir das informações extraídas. Com esse modelo, espera-se que os recursos educacionais possam ser recomendados de uma maneira mais eficiente.

\section{Referências}

Casagrande M. F. R., Kozima G., Wilirich R. (2013). “Técnica de Recomendação Baseada em Metadados para Repositórios Digitais Voltados ao Ensino”. In: Anais do $24^{\circ}$ Simpósio Brasileiro de Informática na Educação (SBIE 2013), Campinas-SP, p. 677-686 de Novembro de 2013.

Cazella, S. C., Bhear, P., Schneider, D., Silva, K.K., Freitas, R. (2012)“Desenvolvendo um Sistema de Recomendação de OAs baseado em Competências para a Educação: relato de experiências." In: Anais do $23^{\circ}$ Simpósio Brasileiro de Informática na Educação (SBIE 2012) Rio de Janeiro, 26-30 de Novembro de 2012. 
Fatemi, M., Tokarchuk, L. (2013) "A Community Based Social Recommender System for individuals \& groups", in: ASE/IEEE International Conference on Social Computing (SocialCom), Washington, DC, pp. 351-356.

Jalali, S. e Wohlin, C. (2012) "Systematic literature studies: database searches vs. backward snowballing," in Proceedings of the ACMIEEE international symposium on Empirical software engineering and measurement, New York, NY, USA, pp. 29-38.

Kim, H.N., Ji, A.T., Ha, I., Jo, G.S. (2010) "Collaborative filtering based on collaborative tagging for enhancing the quality of recommendations", in Electronic Commerce Research and Applications 9 (1) 73-83.

Kitchenham, B. (2004). Procedures for performing systematic reviews. Technical Report, TR/SE-0401, Department of Computer Science, Keele University, UK.

Kitchenham, B. A. and Charters, S. (2007) "Guidelines for performing Systematic Literature Reviews in Software Engineering," Keele University and University of Durham, EBSE Technical Report Version 2.3.

Kitchenham, B., Dag, I.K., Sjøberg, O. P., Brereton, D. B., Dybå, T., Höst, M., Pfahl, D. e Runeson, P. (2010). "Can we evaluate the quality of software engineering experiments?" In: Proceedings of the 2010 ACMIEEE International Symposium on Empirical Software Engineering and Measurement (ESEM 2010), Bolzano-Bozen, Italy, September 16 to 17, 2010. New York: ACM Press, pp. 2:1-2:8.

Park, D.H., Kim, H.K., Choi, I.Y., Kim, J.K. (2012) "A literature review and classification of recommender Systems research", Expert Systems with Applications 39 10059-10072.

Pereira, C. K., Campos, F., Ströele, V., David, J. M. N., Braga, R. (2014) "Extração de Características de Perfil e de Contexto em Redes Sociais para Recomendação de Recursos Educacionais" In: Anais do $25^{\circ}$ Simpósio Brasileiro de Informática na Educação (SBIE 2014) Dourados MS, 03-06 de Novembro de 2014.

Petersen, K. (2011). Measuring and predicting software productivity: A systematic map and review. Information and Software Technology, vol. 53, no. 4, pp. 317-343.

Prikladnicki, R. and Audy, J. L.N. (2010). Process models in the practice of distributed software development: A systematic review of the literature. Information and Software Technology, vol. 52, no. 8, pp. 779-791.

Quijano-Sánchez, L., Díaz-Agudo, B., Recio-García, J. A. (2014) “Development of a group recommender application in a Social Network" in Knowledge-Based Systems.

Rafaeli, S., Dan-Gur, Y., Barak, M. (2005) "Social recommender systems: Recommendations in support of E-learning" in International Journal of Distance Education Technologies, 3 (2), pp. 30-47.

Ribeiro, F. A. A.; Fonseca, L. C. C.; Freitas, M. S. (2013) Recomendando Objetos de Aprendizagem a partir das hashtags postadas no Moodle. In: II CBIE Congresso Brasileiro de Informática na Educação, Unicamp, Campinas, Brasil. 